\title{
ALMOST SURE CONVERGENCE FOR END SEQUENCES AND ITS APPLICATION TO $M$ ESTIMATOR IN LINEAR MODELS
}

\author{
Xin Deng, Shijie Wang, Rui Wang, Xiujuan Xie and Xuejun Wang
}

Abstract. In the paper, an almost sure convergence result for weighted sums of extended negatively dependent random variables is obtained. By using the almost sure convergence result, we further study the strong consistency of $M$ estimator of the regression parameter in linear models based on extended negatively dependent random errors under some mild conditions.

Mathematics subject classification (2010): 62F12, 60F15.

Keywords and phrases: Almost sure convergence, strong consistency, $M$ estimator, extended negatively dependent random error.

\section{REFERENCES}

[1] X. R. Chen, L. C. ZhaO, Strong consistency of $M$-estimates of multiple regression coefficients, Systems Science and Mathematical Sciences, 8 (1), 82-87, 1995.

[2] X. R. Chen, L. C. ZhaO, M-methods in Linear Model, Shanghai: Scientific and Technical Publishers, 1996.

[3] Y. Chen, A. CHEn, K. W. NG, The strong law of large numbers for extend negatively dependent random variables, Journal of Applied Probability, 47, 908-922, 2010.

[4] Y. Chen, K. C. YuEn, K. W. NG, Precise large deviations of random sums in presence of negative dependence and consistent variation, Methodology and Computing in Applied Probability, 13 (4), 821-833, 2011.

[5] J. R. Collins, V. D. Szatmari, Maximal asymptotic biases of $M$ estimators of location with preliminary scale estimates, Communications in Statistics - Theory and Methods, 33 (8), 1877-1866, 2004.

[6] C. DJalil, C. Didier, On the strong consistency of asymptotic M Estimators, Journal of Statistical Planning and Inference, 137, 2774-2783, 2007.

[7] X. M. HE, Q. M. SHAO, A general bahadur representation of $M$ Estimator and its application to linear regression with nonstochastic designs, The Annals of Statistics, 24 (6), 2608-2630, 1996.

[8] P. Georgios, Application of $M$ estimators to cross-section effect models, Communications in Statistics-Simulation and Computation, 34 (3), 601-616, 2005.

[9] T. C. HU, K. L. WANG, A. Rosalsky, Complete convergence theorems for extended negatively dependent random variables, Sankhyā A: The Indian Journal of Statistics, 77 (1), 1-29, 2015.

[10] P. J. HubER, Robust regression: asymptotics, conjectures and Monte Carlo, The Annals of Statistics, 1 (5), 799-821, 1973.

[11] L. LiU, Precise large deviations for dependent random variables with heavy tails, Statistics and Probability Letters, 79, 1290-1298, 2009.

[12] L. LIU, Necessary and sufficient conditions for moderate deviations of dependent random Variables with heavy tails, Science in China Series A: Mathematics, 53 (6), 1421-1434, 2010.

[13] E. L. Lehmann, Some concepts of dependence, The Annals of Mathematical Statistics, 37, 1137$1153,1966$.

[14] K. JoAg-Dev, F. Proschan, Negative association of random variables with applications, The Annals of Statistics, 11 (1), 286-295, 1983. 
[15] D. H. Qiu, P. Y. Chen, R. G. Antonini, A. Volodin, On the complete convergence for arrays of rowwise extended negatively dependent random variables, Journal of the Korean Mathematical Society, 50 (2), 379-392, 2013.

[16] S. SeIJA, T. SARA, O. HANnU, Symmetrized M estimators of multivariate scatter, Journal of Multivariate Analysis, 98, 1611-1629, 2007.

[17] A. T. SHEN, Some strong limit theorems for arrays of rowwise negatively orthant-dependent random variables, Journal of Inequalities and Applications, Volume 2011, Article ID 93, 10 pages, 2011a.

[18] A. T. SHEN, Probability inequalities for END sequence and their applications, Journal of Inequalities and Applications, Volume 2011, Article ID 98, 12 pages, 2011b.

[19] A. T. SHEN, On the strong convergence rate for weighted sums of arrays of rowwise negatively orthant dependent random variables, RACSAM, 107 (2), 257-271, 2013.

[20] A. T. SHEN, On asymptotic approximation of inverse moments for a class of nonnegative random variables, Statistics - A Journal of Theoretical and Applied Statistics, 48 (6), 1371-1379, 2014.

[21] A. T. SHen, Y. ZHANG, A. Volodin, Applications of the Rosenthal-type inequality for negatively superadditive dependent random variables, Metrika, 78 (3), 295-311, 2015.

[22] S. H. SUNG, Complete convergence for weighted sums of negatively dependent random variables, Statistical Papers, 53, 73-82, 2012.

[23] A. Volodin, On the Kolmogorov exponential inequality for negatively dependent random variables, Pakistan Journal of Statistics, 18, 249-254, 2002.

[24] S. J. WANG, W. S. WANG, Precise large deviations for random sums of END real-valued random variables with consistent variation, Journal of Mathematical Analysis and Applications, 402, 660667, 2013.

[25] X. J. WANG, Z. Y. SI, Complete consistency of the estimator of nonparametric regression model under ND sequence, Statistical Papers, 56 (3), 585-596, 2015.

[26] X. J. WANG, S. J. WANG, S. H. Hu, J. M. Ling, Y. F. WEI, On complete convergence of weighted sums for arrays of rowwise extended negatively dependent random variables, Stochastics - An International Journal of Probability and Stochastic Processes, 85 (6), 1060-1072, 2013.

[27] X. J. WANG, X. Q. LI, S. H. HU, X. H. WANG, On complete convergence for an extended negatively dependent sequence, Communications in Statistics - Theory and Methods, 43 (14), 2923-2937, 2014.

[28] X. J. Wang, L. L. Zheng, C. XU, S. H. Hu, Complete consistency for the estimator of nonparametric regression models based on extended negatively dependent errors, Statistics: A Journal of Theoretical and Applied Statistics, 49 (2), 396-407, 2015.

[29] Q. Y. WU, Strong consistency of $M$ estimator in linear model for negatively associated samples, Journal of Systems Science and Complexity, 19 (4), 592-600, 2006 a.

[30] Q. Y. Wu, Probability Limit Theory for Mixing sequences, Science Press of China, Beijing, 2006b.

[31] Q. Y. WU, Y. Y. JIANG, The strong consistency of M estimator in linear model for negatively dependent random samples, Communications in Statistics - Theory and Methods, 40, 476-491, 2011.

[32] Y. F. WU, M. GuAN, Convergence properties of the partial sums for sequences of END random variables, Journal of the Korean Mathematical Society, 49 (6), 1097-1110, 2012.

[33] Y. F. WU, M. O. CABRERA, A. Volodin, Complete convergence and complete moment convergence for arrays of rowwise END random variables, Glasnik Matematicki, 49 (69), 449-468, 2014.

[34] S. C. YAnG, Strong consistency of M estimator in linear model, Acta Mathematica Sinica, 45 (1), 21-28, 2002.

[35] W. Z. YANG, H. Y. XU, L. Chen, S. H. Hu, Complete consistency of estimators for regression models based on extended negatively dependent errors, Statistical Papers, DOI 10.1007/s00362-0160771-x, in press, 2016.

[36] H. ZAREI, H. JABBARI, Complete convergence of weighted sums under negative dependence, Statistical Papers, 52, 413-418, 2011. 